\title{
CORRIGENDUM
}

\section{Are there roles for observational database studies and structured quantification of expert opinion to answer therapy controversies in transplants?}

\author{
RP Gale, M Eapen, B Logan, M-J Zhang and HM Lazarus
}

Bone Marrow Transplantation (2009) 43, 743; doi:10.1038/bmt.2009.72

Correction to: Bone Marrow Transplantation (2009) 43, 435-446. doi:10.1038/bmt.2008.447

Since the publication of their paper, the authors have identified three errors and updates and would like to make the following amendments:

In the second paragraph of page 2, the reference to the Center for International Blood and Marrow Research (CIBMTR) should instead refer to the Blood and Marrow Transplant Clinical Trials Network (BMT CTN).

The sentence beginning on line 11 of the right-hand column of page 9 should now read: 'Finally, an analysis from the CIBMTR comparing auto- and allo-transplants also found no survival benefit.' (Seshadri T, Keating A. Is there a role for autotransplants in AML in first remission? Biol Blood Marrow Transplant 2008 Jan;15(1 Suppl):17-20.)

In the Acknowledgments section, the fifth sentence should start: 'The late Dr Mortimer Bortin along with Dr Alfred Rimm and their colleagues...'
The authors would also like to add the following note to their findings, reported at the end of the third paragraph on page 6 :

'There are nine randomized trials comparing blood and bone marrow grafts from related donor siblings. Meta-analyses of these trials point to differential effects in early and advanced leukemia, and an impact of post-transplant immune suppression on outcomes. (Bensinger W, Stem Cell Trialists' Collaborative Group 2006. Individual patient data meta-analysis of allogeneic peripheral blood stem cell transplant vs bone marrow transplant in the management of hematological malignancies: indirect assessment of the effect of day 11 methotrexate administration. Bone Marrow Transplant 2006; 38: 539-546.)'

The authors apologize for any inconvenience these errors may have caused. 